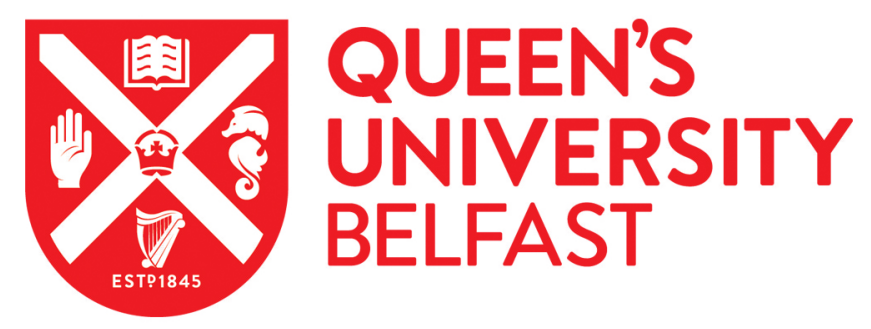

\title{
A Virtual Inspection Technique for Assessing the Dimensional Accuracy of Forged Compressor Blades Using FE Modeling and CMM Inspection
}

Makem, J., Ou, H., Armstrong, C., Rennie, A., \& Nikov, S. (2008). A Virtual Inspection Technique for Assessing the Dimensional Accuracy of Forged Compressor Blades Using FE Modeling and CMM Inspection. International Journal of Material Forming, 1(1), 375-378. https://doi.org/10.1007/s12289-008-0073-5

Published in:

International Journal of Material Forming

Document Version:

Peer reviewed version

Queen's University Belfast - Research Portal:

Link to publication record in Queen's University Belfast Research Portal

\section{General rights}

Copyright for the publications made accessible via the Queen's University Belfast Research Portal is retained by the author(s) and / or other copyright owners and it is a condition of accessing these publications that users recognise and abide by the legal requirements associated with these rights.

Take down policy

The Research Portal is Queen's institutional repository that provides access to Queen's research output. Every effort has been made to ensure that content in the Research Portal does not infringe any person's rights, or applicable UK laws. If you discover content in the Research Portal that you believe breaches copyright or violates any law, please contact openaccess@qub.ac.uk. 


\title{
A Virtual Inspection Technique for Assessing the Dimensional Accuracy of Forged Compressor Blades Using FE Modeling and CMM Inspection
}

\author{
J. Makem ${ }^{1}$, H. Ou ${ }^{1}$, C.G. Armstrong ${ }^{1}$, A. Rennie ${ }^{2}$, S. Nikov². \\ ${ }^{1}$ School of Mechanical \& Aerospace Engineering, Queen's University, Belfast, Ashby Building, Stranmillis \\ Road, Belfast, UK. BT95AH. \\ URL: $\underline{w w w . m e . q u b . a c . u k}$ \\ e-mail:jmakem01@qub.ac.uk; h.ou@qub.ac.uk
}

${ }^{2}$ Rolls-Royce plc, UK.

URL: $\underline{w w w . r o l l s-r o y c e . c o m}$

ABSTRACT: This paper presents research for developing a virtual inspection system that evaluates the dimensional tolerance of forged aerofoil blades formed using the finite element (FE) method. Conventional algorithms adopted by modern coordinate measurement processes have been incorporated with the latest freeform surface evaluation techniques to provide a robust framework for the dimensional inspection of FE aerofoil models. The accuracy of the approach had been verified with a strong correlation obtained between the virtual inspection data and coordinate measurement data from corresponding aerofoil components.

Key words: Forging, Finite Element, Coordinate Measurement, Dimensional Inspection.

\section{INTRODUCTION}

The high demand for manufacturing quality and optimum performance of components within the aerospace industry requires parts to adhere to the relevant geometrical and dimensional specifications. Consequently, components are inspected to evaluate their dimensional tolerance. In particular, the dimensional inspection of complex parts comprising free-form geometry, such as aero-engine blades, is becoming increasingly important due to the requirement for higher precision and efficiency. Generally, the objective behind the majority of inspection techniques is to determine if the part meets a set of specific design criteria by assessing the deviation between the manufactured part and the nominal model.

For quality assurance purposes, high precision dimensional measurement techniques are employed to evaluate the dimensional tolerance of compressor blades. The contact inspection process of coordinate measurement is one of the most effective measurement techniques, providing both high accuracy and repeatability. The process employs a computer controlled coordinate measurement machine (CMM) to inspect the part automatically by moving a tactile probe along the work-piece surface, to measure the coordinates of individual contact points.

Compressor blades are manufactured using the closed die hot forging process. The major contributors to dimensional and shape errors in forged aerofoil components are press and die elasticity during forging, material spring back and thermal distortion at the cooling stage [1]. Due to the complexity of the hot forging process, forging design practice is largely based on the trial and error approach [2]. These forging trials employ a direct compensation approach which involves modifying the die shape by a fraction of the measured forging error until the blade dimensions are within tolerance.

The finite element (FE) method has been used successfully to simulate the forging of compressor blades [3]. By accurately predicting material deformation, stress/strain rate distribution and temperature change in hot forging, the $\mathrm{FE}$ method may be used to quantify the forging errors and optimise the die shape, removing the need for experimental testing and thereby reduce the overall development timescales and costs. Consequently, as 
the FE error governs die shape modification, it's necessary to assess the accuracy of the forging errors produced in simulation by comparison with coordinate measurement data from actual aerofoil sections. Thus, to gain an accurate comparison between the FE generated error and that quantified using the CMM, the FE error must be evaluated using a similar approach to that employed by the coordinate measurement process.

This paper presents a virtual inspection approach used for the validation of an FE blade forming simulation. The developed system provides a fully automated tool for error quantification in a virtual environment. A robust framework has been developed incorporating various iterative algorithms to register the $\mathrm{FE}$ model in relation to a CAD nominal and generate inspection data for comparison with CMM measurements on a real part. It will be shown that a strong correlation exists between simulated and real parts, verifying the validity of the approach.

\section{BLADE INSPECTION}

The process of aerofoil inspection via CMM comprises three main stages, including; part localisation, blade section inspection and finally blade parameters analysis [4]. A unique coordinate system can be established by defining six datum points on the blade. The blade sections are then measured using a two stage process involving an initial rough measurement of each section followed by a more accurate assessment of the profile in fine measurement mode. Finally, after the measurement data for each blade section has been correctly aligned with the respective nominal profile, blade parameters and profile tolerances may be evaluated.

\subsection{Part localisation}

The process of localisation, also known as registration, mathematically locates the part prior to inspection by determining a rigid body 3D coordinate transformation between the design coordinate system (DCS) and the measurement coordinate system (MCS). For aerofoil inspection, the 3-2-1 method or six point principle is commonly used to determine the MCS [5]. Firstly, the primary datum plane is determined by three points on the free-form concave surface of the blade, including $K_{1}$ and $K_{5}$ on the root section and $K_{3}$ on the tip section. The secondary datum plane is constructed using the central axis of cylindrical features at each end of the blade, defined by $P_{1}$ and $P_{2}$. Finally, the tertiary datum plane, orthogonal to both datum planes is determined using the last datum point, $K_{P}$, located on the root block. Finally, both coordinate systems are then aligned via rigid body transformation. The position of the datum points on a nominal blade model is shown in figure 1 .

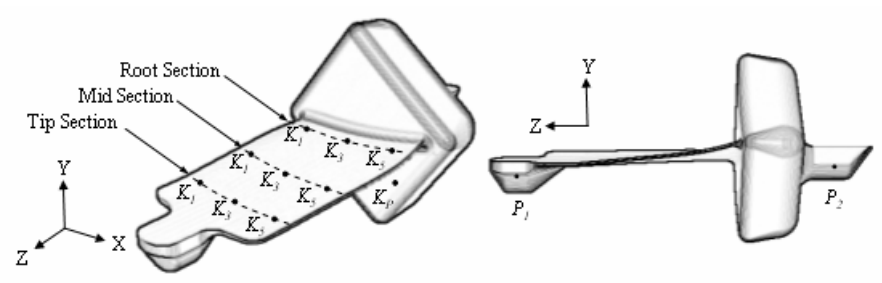

Fig. 1 Datum point locations on nominal blade model.

\subsection{Blade measurement}

The coordinate measurement process employs a contour measurement mode, where by each blade profile was measured at a constant height ( $\mathrm{z}$ coordinate). CMM data is recorded at three sections along the blade for both concave and convex surfaces. Each section is defined by six datum points ( $K$ points) around the profile of the component. A two stage process involving a rough and fine measurement mode is used to evaluate each section.

\subsection{Blade parameters analysis}

Once the profiles of each section have been obtained, a tolerance evaluation algorithm is implemented whereby the convex and concave profiles for each section are positioned with respect to the corresponding nominal profile. The shape tolerance is then represented in the form of a plot comparing the nominal and measured profiles. Also, the thickness, $\delta_{1}$, bow, $\delta_{2}$ and twist, $\delta_{3}$ deviation are evaluated at the relevant $K$ point stations, as shown in figure 2 .

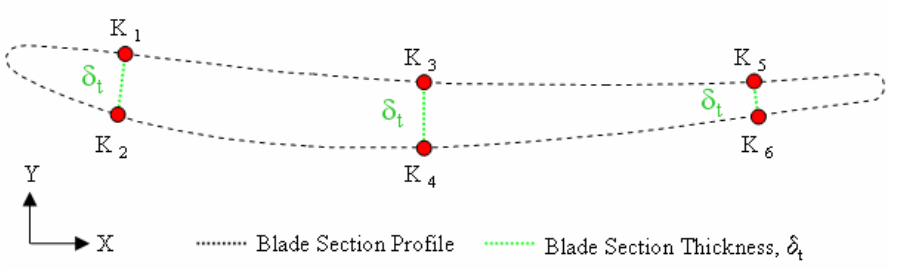

(a)

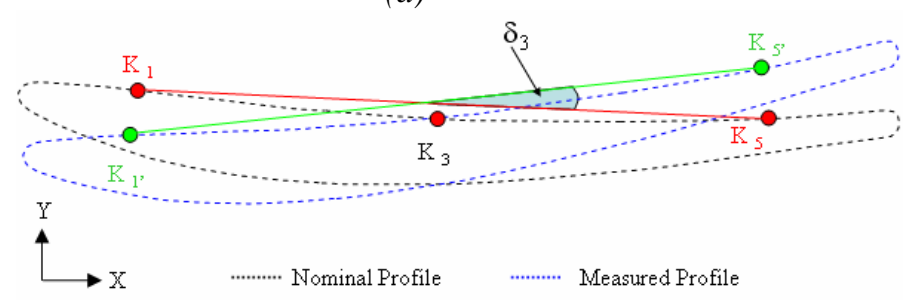

(b)

Fig 2. Measurement of (a) section thickness \& (b) twist error. 
The thickness and bow deviation may be calculated using equations 2 and 3.

$$
\begin{aligned}
& \delta_{t}=\sqrt{\left(x_{K_{c c}}-x_{K_{C V}}\right)^{2}+\left(y_{K_{C C}}-y_{K_{C V}}\right)^{2}} \\
& \delta_{1}=\delta_{t}{ }^{\prime}-\delta_{t} \\
& \delta_{2}=y_{K 3}-y_{K 3^{\prime}}
\end{aligned}
$$

where $\delta_{t}{ }^{\prime}=$ measured thickness and $\delta_{t}=$ nominal thickness.

\section{VIRTUAL INSPECTION SYSTEM}

The main programming languages employed in the development of this software were Python and MATLAB. Figure 3 shows the basic structure of the virtual inspection framework.

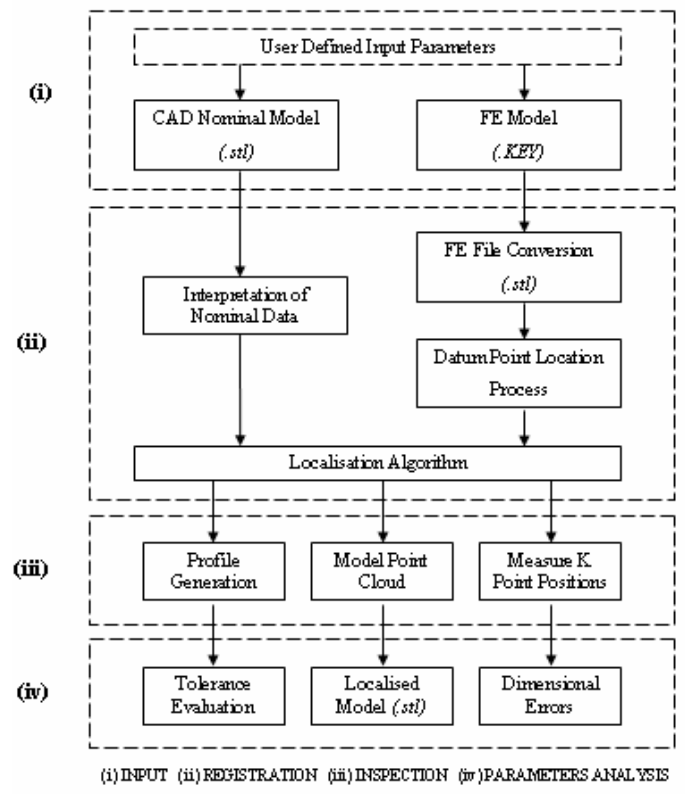

Fig 3. Structure of virtual inspection system.

\subsection{Component registration}

To initiate localisation, datum points were firstly identified on the blade formed from the FE forging simulation. As the initial position of the blade was in roughly close proximity to the nominal coordinate system due to constraints applied in the FE simulation, no preliminary transformation process was required. An initial estimate of the position of datum points $K_{1}, K_{3} \& K_{5}$ shown in figure 1 were found via interpolation from profiles generated by intersecting the FE mesh at the tip and root sections. Subsequently, using the rules of orthogonality and sequence for datum frame construction [6], the secondary datum was established by estimating the location of $P_{1}$ and $P_{2}$. This was achieved by fitting the nodal coordinates situated on each cylindrical surface according to the least squares principal. Consequently, after identifying the respective point clouds using a shape recognition algorithm, each dataset was submitted to the least squares calculation which yielded the coordinates of the datum points on the respective cylinder axes.

After locating the position of five datum points, the normal vectors, $\vec{p}^{\prime}, \vec{s}^{\prime}$ and $\vec{t}^{\prime}$ of the respective datum planes in the MCS were determined using equations $4-6$.

$$
\begin{aligned}
& \vec{p}^{\prime}=\left(K_{5}-K_{1}\right) \times\left(K_{3}-K_{1}\right) /\left|\left(K_{5}-K_{1}\right) \times\left(K_{3}-K_{1}\right)\right| \\
& \vec{s}^{\prime}=\left(P_{2}-P_{1}\right) \times \vec{p}^{\prime} /\left|\left(P_{2}-P_{1}\right) \times \vec{p}^{\prime}\right| \\
& \vec{t}^{\prime}=\left(\vec{p}^{\prime} \times \vec{s}^{\prime}\right) /\left|\vec{p}^{\prime} \times \vec{s}^{\prime}\right|
\end{aligned}
$$

According to the sequence rule in datum setting, the first stage of the localisation involves aligning the components primary datum plane with that of the nominal. This was achieved by aligning $\vec{p}$ with $\vec{p}$, the direction cosine of the primary datum in the DCS. This first transformation was decided by a translation and two rotations in sequence. The second phase involved alignment of the secondary datum planes by rotation about $\vec{p}$. Finally, to align the tertiary datum planes, a single translation, $T$, between the two coordinate frames was applied. The entire transformation is represented by:

$$
X_{1}=R(\beta) R(\theta, \alpha) X_{m}+T
$$

where $R(\theta, \alpha)=$ rotational matrix for angles $\theta$ and $\alpha$ to align $\vec{p}^{\prime}, R(\beta)=$ rotational matrix for angle $\beta$ to align $\vec{s}^{\prime}$ and $X_{m}=$ point on unregistered part.

After the initial registration of the component, the localisation process was repeated until the discrepancy between the datum points on the blade and the corresponding points on the nominal were within a desired tolerance.

\subsection{Comparative analysis and part validation}

Once the part was successfully registered, measurement data was generated by intersecting the aerofoil surface at the respective $K$ point sections using the method described. The section thickness was recorded for each of the nine sets of $K$ points. Also, the bow deviation was recorded at the mid section, as well as the twist deviation at the mid and tip sections. To evaluate the profile tolerance, an additional alignment procedure based on an iterative closest point algorithm [7] was required to fit the relative profile data to the respective nominal curve. 


\section{RESULTS and DISCUSSION}

A contour plot displaying the results of the localisation process is shown in figure 4 .

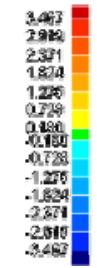

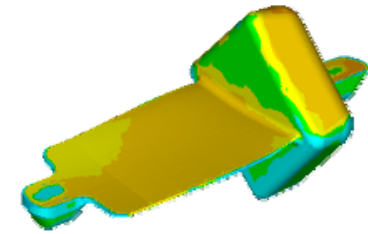

(a) Before Localisation

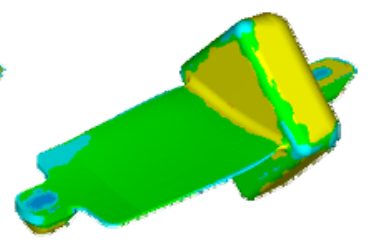

(b) After Localisation
Fig. 4 Deviation between Nominal \& Forged Component

The registration algorithm effectively locates the blade using the iterative six point approach. The deviation between the nominal and the FE model has been dramatically reduced in this area.

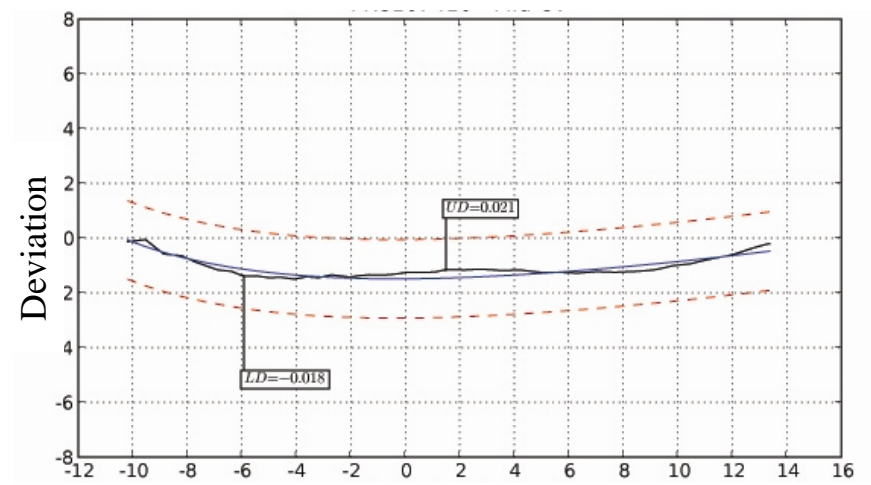

(a) Horizontal Axis

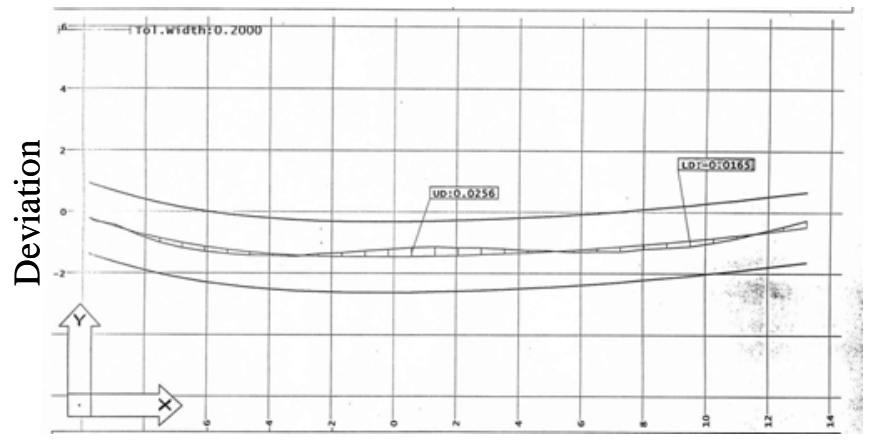

(b) Horizontal Axis

Fig. 5 Comparison between (a) FE and (b) CMM Mid Convex Profile Error

A strong correlation exists between the FE results measured using the virtual system and the actual form measurements. In particular, the data recorded at the mid convex section displays the strongest correlation with a discrepancy of less than $18 \%$ between the maximum profile deviations, as shown in figure 5. Generally, only small discrepancies between the virtual and actual CMM results for both twist and bow deviation were apparent. The virtual measurement of bow deviation differed by approximately $0.01 \mathrm{~mm}$ in comparison the CMM result, whereas a maximum difference of $0.2^{\circ}$ in twist deviation was recorded at the tip section.

In terms of the thickness deviation measurements, values recorded at the root and mid sections are reasonably consistent with CMM values. However, slightly larger discrepancies are apparent at the tip which may be attributed to the inability of the FE method to accurately simulate the deformation in this region.

\section{CONCLUSIONS}

The virtual inspection system provides a complete, robust procedure for the dimensional inspection of forged aerofoil blade models formed using the finite element method. The localisation process used by the system accurately registers the component with the nominal model. The inspection and parameters analysis modules incorporate various iterative algorithms and the latest evaluation techniques to successfully quantify the forging tolerance to within a high degree of accuracy. The strong correlation between measurements generated from this system and actual CMM measurement data, validates the approach as an effective means to quantify the forging tolerance using the coordinate measurement process in a virtual environment.

\section{ACKNOWLODGENTS}

The authors wish to thank the Engineering and Physical Sciences Research Council ESPRC and Rolls-Royce plc for funding and support of the present research (EP/C004140/1).

\section{REFERENCES}

1. Ou H., Lan J., Armstrong C.G., Price M.A., Walloe S.J., Ward M.J., 'Reduction in post forging errors for aerofoil forging using finite element simulation and optimisation'. Modelling Simul.Mater.Sci.Eng, 14, (2006) 179-193

2. Ou H., Armstrong C.G., Price M.A., 'Die shape optimisation in forging of aerofoil sections'. Journal of Materials Processing Technology, 132, (2003) 21-27

3. Hartley P., Pillinger I., 'Numerical simulation of the forging process'. Comput Methods APPL Mech Eng, 195, (2006) 6676-6690

4. Hsu T., Lai J., Ueng W.E., 'On the development of airfoil section inspection and analysis technique'. Int. Journal of Adv Manuf Technol, 30, (2006) 129-140

5. Hsu T., Lai J., Ueng W.E., Hwang J., 'An iterative coordinate setup algorithm for airfoil blades inspection'. Int J Adv Manuf Technol, 26, (2005) 797-807

6. Huang X., Gu P., 'CAD-model based inspection of sculptured surfaces with datums'. Int Journal of Production Research, 36, (1998) 1351-1367

7. Li Y., Gu P., 'Free-form surface inspection techniques state of the art review'. Journal of Computer-Aided Design, 36, (2004) 1395-1417 